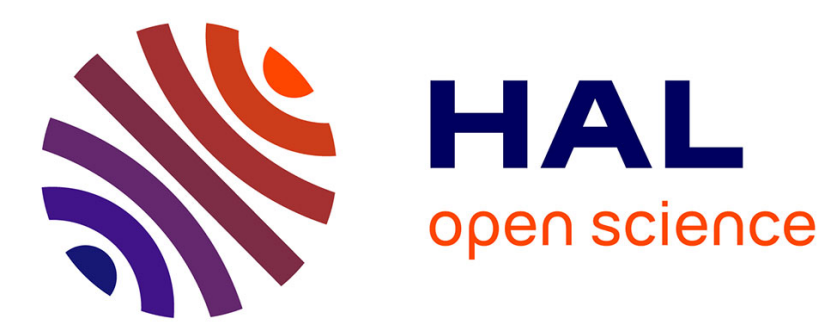

\title{
Probability of detection for GNSS signals with sign transitions
}

\author{
Myriam Foucras, Olivier Julien, Christophe Macabiau, Bertrand Ekambi, \\ Fayaz Bacard
}

\section{> To cite this version:}

Myriam Foucras, Olivier Julien, Christophe Macabiau, Bertrand Ekambi, Fayaz Bacard. Probability of detection for GNSS signals with sign transitions. IEEE Transactions on Aerospace and Electronic Systems, 2016, 52 (3), pp 1296-1308. 10.1109/TAES.2016.140316 . hal-01350127

\section{HAL Id: hal-01350127 https://hal-enac.archives-ouvertes.fr/hal-01350127}

Submitted on 14 Aug 2016

HAL is a multi-disciplinary open access archive for the deposit and dissemination of scientific research documents, whether they are published or not. The documents may come from teaching and research institutions in France or abroad, or from public or private research centers.
L'archive ouverte pluridisciplinaire HAL, est destinée au dépôt et à la diffusion de documents scientifiques de niveau recherche, publiés ou non, émanant des établissements d'enseignement et de recherche français ou étrangers, des laboratoires publics ou privés. 


\title{
Probability of Detection for GNSS Signals With Sign Transitions
}

\author{
Myriam Foucras, Olivier Julien, Christophe Macabiau, Bertrand Ekambi, Fayaz Bacard
}

\begin{abstract}
In Global Navigation Satellite Systems (GNSS) receivers, the acquisition process is the first stage of the signal processing module. It consists of assessing the presence of GNSS signals and providing a rough estimation of the incoming signal parameters: the Doppler frequency and the code delay. However, the presence of bit sign transitions affects receiver performance in signal acquisition detection. This article focuses on the bit transition and its impact on the acquisition performance by providing a general mathematical study and an illustration for two GNSS signals: the global positioning system (GPS) legacy civil signal (L1 C/A) and Galileo E1 open service (OS). This study is led for a terrestrial user in a constraint environment. Furthermore, the presented results are mathematical models of the probability of detection in presence of bit sign transitions (only one potential bit sign transition per integration interval) and potential uncertainties on the Doppler frequency and code delay, these do not results from empirical acquisition of real signals.
\end{abstract}

Index Terms-Acquisition; Bit sign transition; GNSS; Probability of detection

\section{INTRODUCTION}

$\mathrm{G}^{-1}$ LOBAL Navigation Satellite System (GNSS) signals are composed of a carrier modulated by a spreading sequence (or pseudorandom noise, PRN sequence) and a binary navigation message. Initially, the GNSS signals were only based on one component (such as GPS L1 C/A [1]) which was used for both data communication and ranging. The new generation of signals (such as GPS L1C [2], GPS L5 [3], Galileo E1 OS and Galileo E5a/b [4]) has two components. One is called the data component, which contains the navigation message and the other is the pilot component or dataless component, not modulated by a navigation data stream [4].

GNSS signal acquisition consists of assessing the presence of the GNSS signals and of providing a rough estimation of the

\footnotetext{
${ }^{M}$ anuscript received August 28, 2014; revised May 6, 2015 and October 20, 2015; released for publication November 12, 2015.

Authors' addresses: M. Foucras, B. Ekambi, F. Bacard ABBIA GNSS Technologies 27 rue Jules Amilhau 31100 Toulouse, France (\{myriam.foucras, bertrand.ekambi, fayaz.bacard $\} @$ abbia.fr); O. Julien and C. Macabiau TELECOM Lab/SIGNAV ENAC 7 avenue Edouard Belin 31055 Toulouse, France (\{ojulien, macabiau\}@ recherche.enac.fr) IEEE Log No. T-AES/201400316
}

incoming signals' parameters: Doppler and code delay. The Doppler frequency is mainly driven by the motion of the satellite, the receiver oscillator clock drift and the user dynamics [5]. In this study, it is assumed that the GNSS receiver is used for terrestrial applications (for example, a car) so the Doppler frequency range is $[-5 ; 5] \mathrm{kHz}$. The PRN code delay is related to the propagation time and hardware components [6].

The classical GNSS signal acquisition method, the serial search, is based on the use of a two-dimensional acquisition grid which should cover the uncertainty on the GNSS signal Doppler and code delay values. To detect the presence of the GNSS signal, the received signal is correlated with a succession of locally generated replicas of the GNSS signal whose parameters (Doppler and code delay) are taken from the acquisition grid. Each point of the grid then sequentially translates into an acquisition detector value and the process continues until the acquisition detector crosses a predefined threshold. For this acquisition method, the absolute value of the incoming Doppler frequency or code delay is not important, the acquisition performance depends on the relative difference between the real value and the point of the grid (called the uncertainty).

It is well known that the presence of a bit transition during the correlation process is detrimental to the signal-to-noise ratio at the correlator output and thus to the detection capability of the GNSS receiver [7]. However, few papers found in the literature investigate the mathematical model of this problem and its impact on the acquisition performance (for instance, [8] for GPS L1 C/A). The present paper intends to propose an extended study of the effect of the bit transition on the acquisition performance, including the new GNSS signals. The motivations behind this investigation are multiple:

- Understand in a general way the effect of bit transitions on the acquisition process (bit transition location, probability of bit transition, bit rate, etc...).

- Study the impact of the bit transition for different GNSS signal structures. To do so, two signals: the legacy GPS L1 C/A and the new Galileo E1 OS are taken as examples.

- Provide the probability to detect the useful signal when considering bit transitions and uncertainties on the Doppler frequency and code delay.

- Provide a theoretical dwell time to detect real signals (with bit sign transitions and random Doppler frequency and code delay) in the context of a terrestrial application and with a given average probability. Like the other 
results, the integration time is chosen so that there is a maximum of one bit sign transition per integration interval.

We have already presented results in [9] for Galileo E1 OS but theoretical and generic results presented here can be applied to any GNSS. The outline of the paper is as follows. The second section reviews the GNSS signal acquisition principle. In this section, the correlator output expressions are developed and the performance study based on a statistical test is presented. The next section is dedicated to the mathematical model of the probability of detection in presence of bit transitions. In the fourth section, always considering the bit transitions, we provide the probability to detect the signal when the estimated parameters are in the "right" cell. This permits to remove the small uncertainty because of the cell width induced by the acquisition grid. The next section applies the previous results to two GNSS signals: GPS L1 C/A and Galileo E1 OS. Results come from an implementation of the previously given model of the probability of detection in presence of bit sign transitions and uncertainties. The last section concludes the paper, giving the remaining main points of interests and results.

\section{ACQUISITION}

Before delving into the details of the acquisition process principle, we present the structure of the received signal. The structure and properties of signals can be completed using Interface Control Document (ICD), for GPS L1 C/A [1] and for Galileo E1 OS [4].

\section{A. GNSS signals' Model}

The signal entering the signal processing part of a GNSS receiver is a combination of GNSS signals, which are emitted by several satellites from potentially multiple constellations, and perturbations (noise, interference, etc.). The signal associated to one satellite is generically composed of the following:

- A carrier, whose frequency depends on the receiver intermediate frequency, denoted $f_{I F}$ and Doppler frequency, denoted $f_{d}$

- A navigation message, denoted $d$, that can be seen as a random sequence of 1 and -1 and which bit duration is denoted $T_{d}$

- A spreading code, denoted $c$ that is specific to each signal. A bit of a spreading code is called "chip" to mark the difference between a useful data bit and a spreading code bit. The spreading code period is denoted $T_{c}$

A known secondary code

Based on this generic model, the received GPS L1 C/A signal at the RF front-end output can be expressed for one satellite as follows as:

$$
\begin{aligned}
r(t) & =A d(t-\tau) c(t-\tau) \cos \left(2 \pi\left(f_{d}+f_{I F}\right) t+\phi_{0}\right) \\
& +n(t)
\end{aligned}
$$

Where:

- $A$ is the amplitude of the incoming signal at the correlator input. In this case, $A$ is related to the signal power $C$ by $A=\sqrt{2 C}$ (to be generic, $A$ can be written $A=\sqrt{\beta C}$ with $\beta=2$ for GPS L1 C/A)
- $\phi_{0}$ is the initial phase of the incoming signal

- $n$ is the incoming noise, which is assumed to be a white noise with centered Gaussian distribution with a constant two-sided power spectral density equal to $N_{0} / 2 \mathrm{dBW} / \mathrm{Hz}$

- $\tau$ is the code delay caused by the transmission and hardware biases

The Galileo E1 OS signal has two (data and pilot) components that are in-phase. Its expression is:

$$
\begin{aligned}
r(t) & =A\left[\begin{array}{c}
d(t-\tau) c_{D}(t-\tau) p_{D}(t-\tau) \\
-c_{2}(t-\tau) c_{P}(t-\tau) p_{P}(t-\tau)
\end{array}\right] \\
& \times \cos \left(2 \pi\left(f_{d}+f_{I F}\right) t+\phi_{0}\right)+n(t)
\end{aligned}
$$

Where:

- $A$ is the amplitude of one of the component of the incoming signal at the correlator input. In this case, $A$ is related to the total signal power $C$ (data+pilot components) by $A=\sqrt{C}$ (and $\beta=1$ ).

- $c_{2}$ is the secondary code on the pilot component. The duration of one secondary code bit is the data bit duration $T_{d}$.

- The spreading codes $c_{x}$ on the data component and on the pilot component are distinguished using a subscript ( $x$ being " $D$ " for Data and " $P$ " for Pilot)

- $p_{x}$ represents the subcarrier modulating the spreading codes for composite binary offset carrier (CBOC) signals [4]. It is different between the data and pilot components.

For Galileo E1 OS, the power of the signal is equally distributed on both components (50\% on each). In this paper, it is assumed that the total received power is the same for GPS L1 C/A and for Galileo E1 OS signals (considering both components).

Up to the section IV, only one component is considered. The structure and properties of the data component are the same as those of the pilot component and the secondary code has an effect similar to that of a data sequence during the acquisition stage.

\section{B. GPS L1 C/A and Galileo E1 OS Signals Characteristics}

The characteristics of the civil GPS and Galileo signals in

\begin{tabular}{|c|c|c|c|}
\hline \multirow{2}{*}{ Signal } & \multirow{2}{*}{ GPS L1 C/A } & \multicolumn{2}{|c|}{ Galileo E1 OS } \\
\hline & & Data & Pilot \\
\hline $\begin{array}{l}\text { Spreading } \\
\text { modulation }\end{array}$ & BPSK & $\begin{array}{c}\mathrm{CBOC}(6,1, \\
\left.1 / 11,{ }^{\prime}+'\right)\end{array}$ & $\begin{array}{c}\mathrm{CBOC}(6,1, \\
1 / 11,-')\end{array}$ \\
\hline $\begin{array}{l}\text { Code } \\
\text { frequency }\end{array}$ & $1.023 \mathrm{MHz}$ & \multicolumn{2}{|c|}{$1.023 \mathrm{MHz}$} \\
\hline $\begin{array}{l}\text { Spreading } \\
\text { code length }\end{array}$ & $\begin{array}{l}1023 \text { chips } \\
T_{c}=1 \mathrm{~ms}\end{array}$ & \multicolumn{2}{|c|}{$\begin{array}{l}4092 \text { chips } \\
T_{C}=4 \mathrm{~ms}\end{array}$} \\
\hline Bit rate & $50 \mathrm{bit} / \mathrm{s}$ & $\begin{array}{c}\text { Data } \\
250 \mathrm{bit} / \mathrm{s}\end{array}$ & $\begin{array}{c}\text { Secondary code } \\
250 \mathrm{bit} / \mathrm{s}\end{array}$ \\
\hline Bit duration & $T_{d}=20 \mathrm{~ms}$ & $T_{d}=4 \mathrm{~ms}$ & $T_{c_{2}}=4 \mathrm{~ms}$ \\
\hline
\end{tabular}
the L1 band are given in TABLE I.

TABLE I

GPS L1 C/A AND GALILEO E1 OS SIGNAL TECHNICAL CHARACTERISTICS

It is worth noting that:

- For GPS L1 C/A, the data bit duration is 20 times longer than spreading code period. This implies that a data bit 
sign transition occurs only once every twenty spreading code periods with a probability of $50 \%$.

- For Galileo E1 OS, the spreading code period is the same as the duration of a data or secondary code bit. This implies that a bit sign transition (data bit and/or secondary code bit) occurs at each spreading code period with a probability of $50 \%$.

\section{Acquisition Principle}

For now, the acquisition principle is presented when one component is considered. The acquisition process is based on a correlation operation. A local replica of the received signal (composed exclusively of the carrier, the spreading code and optionally the secondary code) is correlated with the received signal. By generating a set of local replicas that take all possible values for the carrier frequency and spreading code delay of the incoming signal (called as uncertainty space) and using a relevant detector, it is possible to roughly estimate two parameters of the incoming signal: the Doppler frequency and the code delay. One classical acquisition strategy [10] is the serial-search acquisition method, which consists of testing successively many possible (carrier frequency and spreading code delay) couples based on a discretization of the uncertainty space [11]. Let $\Delta_{f}$ be the uncertainty width in the frequency search space and $\Delta_{\tau}$ be the uncertainty width in the code delay search space.

\section{Correlator Outputs in Absence of Data}

Let us study the acquisition process in an ideal case, which assumes that there is no data in (1). This is equivalent to assume that $d$ is constant and equal to 1 .

The general structure of the sequential acquisition is depicted in Fig. 1 and [12].

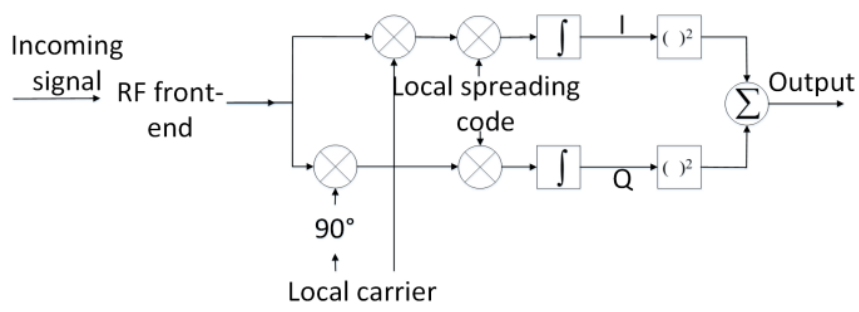

Fig. 1: Block diagram of the serial search acquisition

The local code delay and carrier replicas are modeled as:

- $\quad c(t-\hat{\tau})$ where $\hat{\tau}$ is the local code delay

- $\cos \left(2 \pi\left(f_{I F}+\widehat{f}_{d}\right) t\right)$, where $\widehat{f}_{d}$ is the local Doppler frequency

Assume that $\left[T_{0}+k T_{I} ; T_{0}+(k+1) T_{I}\right]$ is the coherent integration interval (with $T_{I}$ as an integer multiple of the spreading code period and shorter or equal to a bit duration $T_{I} \leq T_{d}$ ) and that parameters of the processed signal and the local replica are constant during the correlation operation such that the code delay error $\varepsilon_{\tau}$ and the Doppler frequency error $\varepsilon_{f}$ are constant and the carrier phase error at the beginning of the correlation process is $\varepsilon_{\phi_{0}}$. Then, it is possible to show [6] that the in-phase correlator output, denoted $I(k)$ in Fig. 1 can be modeled by:

$$
\begin{aligned}
I(k) & =\frac{A}{2} R\left(\varepsilon_{\tau}\right) \cos \left(\pi \varepsilon_{f} T_{I}+\varepsilon_{\phi_{0}}(k)\right) \operatorname{sinc}\left(\pi \varepsilon_{f} T_{I}\right) \\
& +n_{I}(k)
\end{aligned}
$$

where

- $n_{I}$ represents the noise at the in-phase correlator output, which is assumed to follow a centered Gaussian distribution whose variance is $\sigma^{2}=\frac{N_{0}}{4 T_{I}}[13]$

- $R$ is the correlation function between the local and the incoming spreading sequence (including the subcarrier)

The quadrature-phase correlator output, represented as $Q$ in Fig. 1 is:

$$
\begin{aligned}
Q(k) & =\frac{A}{2} R\left(\varepsilon_{\tau}\right) \sin \left(\pi \varepsilon_{f} T_{I}+\varepsilon_{\phi_{0}}(k)\right) \operatorname{sinc}\left(\pi \varepsilon_{f} T_{I}\right) \\
& +n_{Q}(k)
\end{aligned}
$$

where $n_{Q}$ is the noise at the quadrature correlator output which has the same distribution as $n_{I}$ but is independent from it.

\section{E. Acquisition as a Detection Problem}

The acquisition process can be seen as a detection problem because the purpose of the acquisition is to detect whether a signal from a given satellite is present at the receiver level [8]. The usual acquisition detector is expressed as:

$$
T=\sum_{k=1}^{K}\left(\frac{I^{2}(k)}{\sigma^{2}}+\frac{Q^{2}(k)}{\sigma^{2}}\right)
$$

where $K$ is the number of non-coherent summations. The duration $K T_{I}$ is the dwell time in an acquisition bin.

The detector is then compared to a predefined threshold $T_{h}$ to declare whether the signal is present. In this context, false alarms and missed detection are possible. The probability of false alarm $P_{f a}$ is generally small and in this paper, fixed to $P_{f a}=10^{-3}$, as in [14].

The detection problem can be seen as a hypothesis test with the following hypothesis:

- The null hypothesis $H_{0}$ assumes that the useful signal is not present in the incoming signal or that the investigated cell of the acquisition grid is not the correct one. Because of the correlation properties of the GNSS spreading codes, these two cases can be grouped. In other words, it can be assumed that there is only noise at the correlator outputs.

- The alternative hypothesis $H_{1}$ assumes that the useful signal is present and that the investigated cell of the acquisition grid is the correct one.

The acquisition detection test can be re-written as:

$$
H_{0}: T<T_{h} \text { against } H_{1}: T \geq T_{h}
$$

1) Hypothesis $H_{0}$

Let us consider the null hypothesis and determine the detection threshold knowing the desired probability of false alarm. Under this hypothesis, the correlator outputs (3) and (4) can be seen as only noise. Then $I$ and $Q$ have centered Gaussian distribution whose variance is equal to $\sigma^{2}$. The division of $I^{2}$ and $Q^{2}$ by $\sigma^{2}$ permits us to normalize the correlator outputs to have unit Gaussian random variables. Then, the detector under the null hypothesis, $T_{H_{0}}$, follows a $\chi^{2}$ distribution with $2 K$ degrees of freedom whose cumulative distribution function is denoted $F_{\chi^{2}}$. 


$$
T=\sum_{k=1}^{K}\left(\frac{I^{2}(k)}{\sigma^{2}}+\frac{Q^{2}(k)}{\sigma^{2}}\right) \sim_{H_{0}} \chi^{2}(2 K)
$$

For a desired probability of false alarm, $P_{f a}$, the detection threshold can be easily deduced:

$$
\begin{aligned}
& P_{f a}=P_{H_{0}}\left(\mathrm{~T}>T_{h}\right)=1-F_{\chi^{2}(2 K)}\left(T_{h}\right) \\
& \text { 2) Hypothesis } H_{1}
\end{aligned}
$$

Let us assume that the useful signal is present and study the of detecting the presence of the GNSS signal. It can be assumed that $\varepsilon_{f}$ and $\varepsilon_{\tau}$ are small (within the acquisition cell uncertainty space). The distributions of the correlator outputs are given by:

$$
\begin{aligned}
& I(k) \sim \mathcal{N}\left(E[I(k)], \sigma^{2}\right) \\
& Q(k) \sim \mathcal{N}\left(E[Q(k)], \sigma^{2}\right)
\end{aligned}
$$

Where $E[$.$] is the expectation operator. The detector is then$ characterized by the following:

$$
T=\sum_{k=1}^{K}\left(\frac{I^{2}(k)}{\sigma^{2}}+\frac{Q^{2}(k)}{\sigma^{2}}\right) \sim_{H_{1}} \chi^{2}\left(2 K, K \lambda_{0}\right)
$$

The distribution of the acquisition detector becomes a noncentral $\chi^{2}$ distribution. Assuming that the parameters of $I(k)$ and $Q(k)$ remain the same during the dwell time in one acquisition grid cell, the non-centrality parameter is equal to $K \times \lambda_{0}$ where

$$
\lambda_{0}=\beta \frac{C}{N_{0}} \times T_{I} R^{2}\left(\varepsilon_{\tau}\right) \operatorname{sinc}^{2}\left(\pi \varepsilon_{f} T_{I}\right)
$$

Knowing the distribution of the detector, it is possible to evaluate the probability of detection, $P_{d_{0}}$ :

$$
P_{d_{0}}=P_{H_{1}}\left(\mathrm{~T}>T_{h}\right)=1-F_{\chi^{2}\left(2 K, K \times \lambda_{0}\right)}\left(T_{h}\right)
$$

For relatively small Doppler frequency error as in this study, it can be seen from this expression that the probability of detection depends on the following:

- The received carrier-to-noise ratio $C / N_{0}$ (the higher, the better)

- The integration time $T_{I}$ (the longer, the better)

- The Doppler frequency and code delay errors $\varepsilon_{f}$ and $\varepsilon_{\tau}$ (the smaller, the better)

- The number of noncoherent summations $K$ (the higher, the better)

As an example, a representation of the acquisition detector for each couple of the acquisition grid is given in Fig.2 for GPS L1C/A.

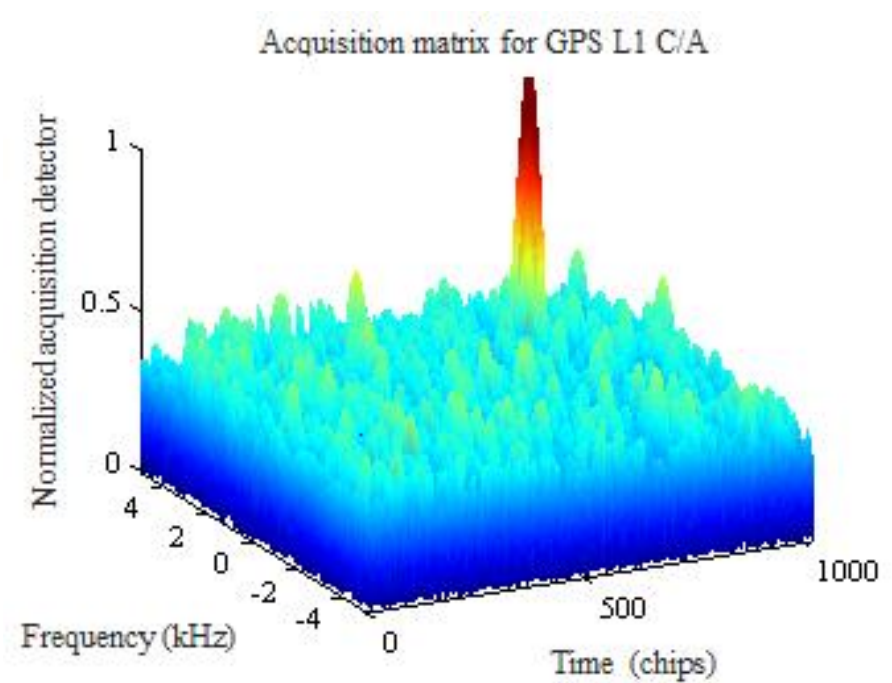

Fig. 2: Acquisition matrix output from serial search acquisition

\section{F. Acquisition parameters}

The choice of the acquisition cell size comes from a compromise between minimizing $\varepsilon_{f}$ and $\varepsilon_{\tau}$ to have a better probability of detection and minimizing the number of cells to reduce the acquisition time. In the context of this paper, it was decided to follow the recommendations given in [14]:

$$
-\left|\Delta_{f}\right| \leq \frac{1}{2 T_{I}}
$$

- $\left|\Delta_{\tau}\right| \leq \frac{1}{2}$ chip for GPS L1 C/A and in an equivalent way $\left|\Delta_{\tau}\right| \leq \frac{1}{6}$ chip for Galileo E1 OS.

Such a cell size results in a worst-case degradation in terms of equivalent $C / N_{0}$ at the correlator output of approximately $3.5 \mathrm{~dB}$, which comes from the possibility that the actual incoming parameters are at the edge of the correct acquisition cell.

\section{BIT SIGN TRANSITION}

In this paper, it is assumed that the acquisition is done in cold start, meaning that there is no a-priori information used by the receiver. In this context, the location of the data bit transition in the correlation interval is unknown. Considering this case, the correlation operation, and thus the detection performance results, can become radically different. The following section develops the associated mathematical model and describes the impact of bit sign transitions on the acquisition of GNSS signals.

Before looking at the problem of the bit sign transition on the acquisition performance, let us define the terminology used in the following:

- A bit transition is defined as the transition between 2 consecutive bits of the useful data sequence or secondary code

- During a bit transition, a sign transition can occur. Assuming that the data sequence is random and each bit value is independent from the previous one, a sign transition occurs with a probability of $50 \%$. 


\section{A. $\quad$ Correlator outputs in presence of bit sign transition}

The correlator output model can be established by considering that a bit sign occurs. Let us assume that the following:

- The correlation time is assumed to be shorter than the data bit duration $T_{I} \leq T_{d}$; then, at the maximum, one bit sign transition can occur within the correlation interval

- $\quad$ The correlation interval is chosen to be $\left[0 ; T_{I}\right]$

- A bit sign transition occurs at $t_{0}$ with $0 \leq t_{0} \leq T_{I}$. For example,

$$
d(t)=\left\{\begin{array}{l}
+1, t \in\left[0 ; t_{0}\right] \\
-1, t \in\left[t_{0} ; T_{I}\right]
\end{array}\right.
$$

Fig. 3 illustrates the previously presented assumptions. Here, two bit transitions are represented but there is only one bit sign transition between the bit $n$ and the bit $n+1$.

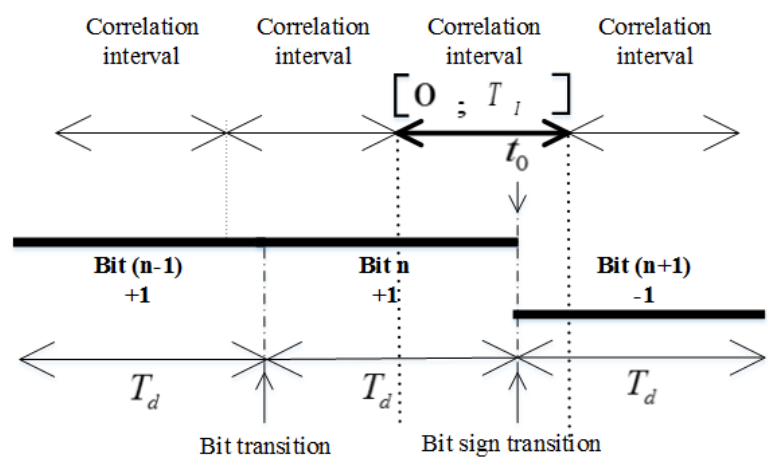

Fig. 3: Bit sign transition scheme

With these assumptions, let us evaluate the in-phase correlator output:

$$
\begin{aligned}
I_{t_{0}}(k) & =\frac{1}{T_{I}} \int_{0}^{t_{0}} r^{\prime(t-\tau)} c(t-\hat{\tau}) \cos \left(2 \pi\left(f_{I F}+\widehat{f_{d}}\right) t\right) d t \\
& -\frac{1}{T_{I}} \int_{t_{0}}^{T_{I}} r^{\prime}(t-\tau) c(t-\hat{\tau}) \cos \left(2 \pi\left(f_{I F}+\widehat{f_{d}}\right) t\right) d t
\end{aligned}
$$

where $r^{\prime}(t-\tau)$ is the received signal $r(t-\tau)$ without the data bit. Using trigonometric identities $I_{t_{0}}(k)$ becomes:

$$
I_{t_{0}}(k)=\frac{A}{2} R\left(\varepsilon_{\tau}\right)\left[\begin{array}{c}
-\sin \left(\pi \varepsilon_{f} T_{I}+\varepsilon_{\phi_{0}}(k)\right) \frac{\cos \left(\pi \varepsilon_{f} T_{I}\right)}{2 \pi \varepsilon_{f} T_{I}} \\
+\frac{\sin \left(2 \pi \varepsilon_{f} t_{0}+\phi_{0}\right)}{2 \pi \varepsilon_{f} T_{I}}
\end{array}\right]
$$

Similarly, the quadrature correlator output, $Q_{t_{0}}(k)$, in the presence of a bit sign transition at $t_{0}$ is as follows:

$$
\begin{aligned}
& Q_{t_{0}}(k)=\frac{A}{2} R\left(\varepsilon_{\tau}\right)\left[\begin{array}{c}
-\cos \left(\pi \varepsilon_{f} T_{I}+\varepsilon_{\phi_{0}}(k)\right) \frac{\cos \left(\pi \varepsilon_{f} T_{I}\right)}{2 \pi \varepsilon_{f} T_{I}} \\
+\frac{\cos \left(2 \pi \varepsilon_{f} t_{0}+\phi_{0}\right)}{2 \pi \varepsilon_{f} T_{I}}
\end{array}\right] \\
& +n_{Q_{t_{0}}}(k)
\end{aligned}
$$

\section{B. Impact on acquisition when no non-coherent summation is used}

Considering that the acquisition is only based on one set of correlator output $(K=1)$, then in the presence of a data bit transition (in this section, we assume that the bit sign transition occurs within the correlation interval), the normalized acquisition detector, becomes:

$$
T_{t_{0}}=\frac{I_{t_{0}}^{2}(k)}{\sigma^{2}}+\frac{Q_{t_{0}}^{2}(k)}{\sigma^{2}}
$$

Similar to the hypothesis test in Section II.E, under $H_{0}, T_{t_{0}}$ has the same distribution as the $T: \chi^{2}$ distribution with 2 degrees of freedom. Under $H_{1}, T_{t_{0}}$ becomes a noncentral $\chi^{2}$ distribution with 2 degrees of freedom, and the noncentrality parameter is $\lambda_{t_{0}}$ (a function of the instant of the bit sign transition $\left.t_{0}\right)$ :

$$
\begin{aligned}
& \lambda_{t_{0}}=\beta \frac{C}{N_{0}} T_{I} R^{2}\left(\varepsilon_{\tau}\right) \times \\
& \frac{\cos ^{2}\left(\pi \varepsilon_{f} T_{I}\right)+1-2 \cos \left(\pi \varepsilon_{f} T_{I}\right) \cos \left(\pi \varepsilon_{f}\left(T_{I}-2 t_{0}\right)\right)}{\left(\pi \varepsilon_{f} T_{I}\right)^{2}}
\end{aligned}
$$

Fig. 4 represents the noncentrality parameter $\lambda_{t_{0}}$ as a function of the Doppler frequency error and the bit transition instant in a coherent integration time of $1 \mathrm{~ms}$ (GPS L1 C/A) for a received signal power of $27 \mathrm{~dB}-\mathrm{Hz}$.

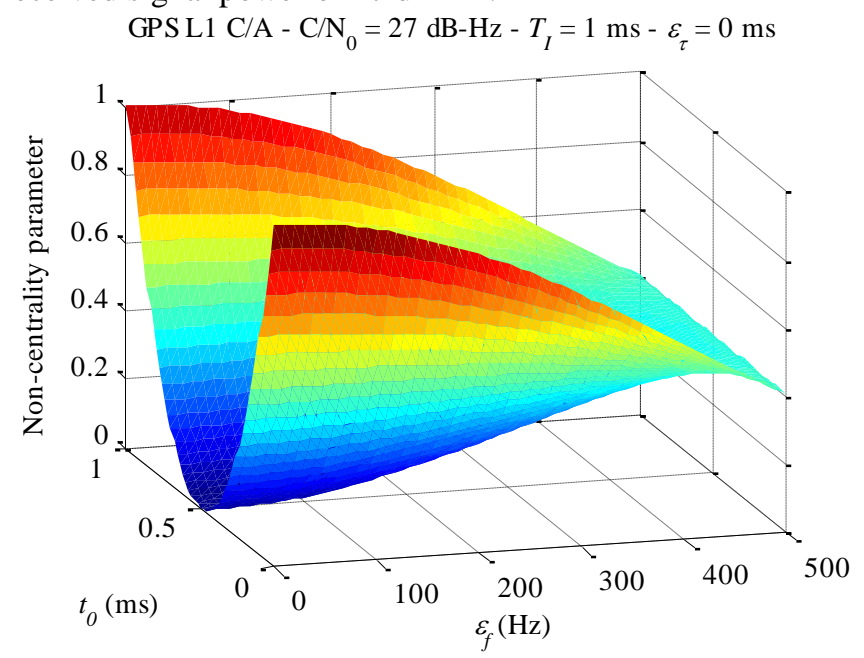

Fig. 4: Non-centrality parameter in presence of a bit sign transition

Fig. 4 and (18) help to show that degradation of the noncentrality parameter $\lambda_{t_{0}}$ with respect to $\lambda_{0}$ depends on the Doppler frequency error $\varepsilon_{f}$ and the location of the bit sign transition $t_{0}$. The worst location for the bit sign transition is in the middle of the correlation interval $\left(t_{0}=T_{I} / 2\right)$. In this case, for $\varepsilon_{f} \neq 0$,

$$
\lambda_{t_{0}}=\beta \frac{C}{N_{0}} T_{I} R^{2}\left(\varepsilon_{\tau}\right) \frac{\left(1-\cos \left(\pi \varepsilon_{f} T_{I}\right)\right)^{2}}{\left(\pi \varepsilon_{f} T_{I}\right)^{2}}
$$

Let us assess the effect of the bit sign transition on the noncentrality parameter $\lambda_{t_{0}}$.In the case of the GPS L1 C/A signal, if $t_{0}$ is a multiple of $T_{I}$, meaning that the bit sign transition occurs at the beginning of the spreading code period, then it is as if no bit sign transition occurred. If $\varepsilon_{f}=0$, then $\lambda_{t_{0}}$ needs to be assessed carefully because $\varepsilon_{f}$ is in the denominator in (18). Replacing $u=\pi \varepsilon_{f} T_{I}$ in (18) and knowing that when $u$ is in the neighborhood of $0, \cos (u)$ can be approximated by $\left(1-\frac{u^{2}}{2}\right)$, it follows that 


$$
\begin{aligned}
\cos ^{2}(u)+1 & =\frac{1+\cos (2 u)}{2}+1 \\
& \approx u \approx 0 \\
& 2-u^{2}=2\left(1-\frac{u^{2}}{2}\right)
\end{aligned}
$$

$$
\begin{aligned}
& 2 \cos (u) \cos \left(u\left(1-\frac{2 t_{0}}{T_{I}}\right)\right) \\
& \approx_{u \approx 0} 2\left(1-\frac{u^{2}}{2}\right)\left(1-\frac{u^{2}}{2}\left(1-\frac{2 t_{0}}{T_{I}}\right)^{2}\right) \\
& \approx_{u \approx 0} 2\left(\left(1-\frac{u^{2}}{2}\right)-\left(1-\frac{u^{2}}{2}\right) \frac{u^{2}}{2}\left(1-\frac{2 t_{0}}{T_{I}}\right)^{2}\right)
\end{aligned}
$$

Then, it follows that around 0 :

$$
\frac{2\left(1-\frac{u^{2}}{2}\right)-2\left(1-\frac{u^{2}}{2}\right)+2\left(1-\frac{u^{2}}{2}\right) \frac{u^{2}}{2}\left(1-\frac{2 t_{0}}{T_{I}}\right)^{2}}{\approx_{u \approx 0}\left(1-\frac{u^{2}}{2}\right)\left(1+4 \frac{t_{0}^{2}}{T_{I}^{2}}-4 \frac{t_{0}}{T_{I}}\right)}
$$

This leads to the following approximation of $\lambda_{t_{0}}$ for $\varepsilon_{f} \approx 0$,

$$
\lambda_{t_{0}} \approx \beta \frac{C}{N_{0}} T_{I} R^{2}\left(\varepsilon_{\tau}\right)\left(1+4 \frac{t_{0}^{2}}{T_{I}^{2}}-4 \frac{t_{0}}{T_{I}}\right)
$$

In this case, if $t_{0}$ is equal to $T_{I} / 2, \lambda_{t_{0}}$ is null.

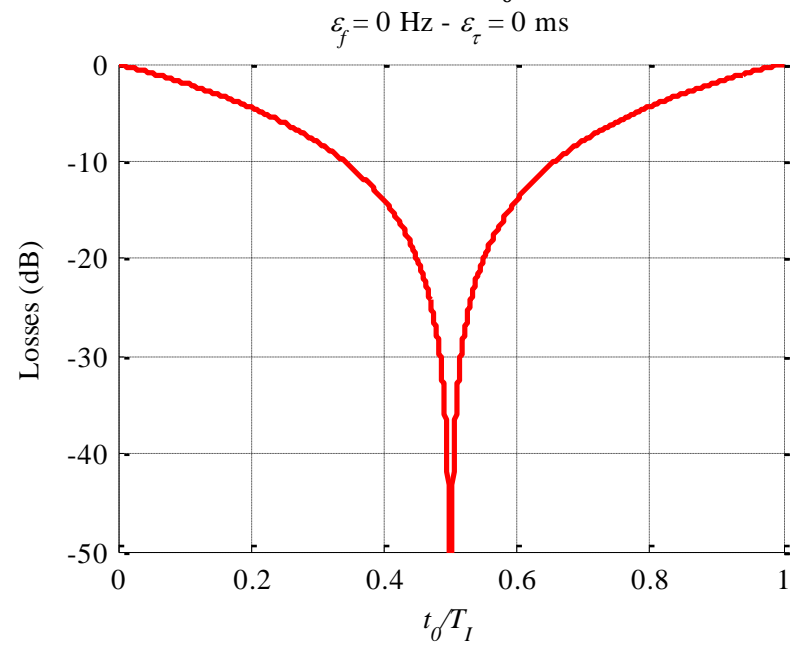

Fig. 5: Losses on the probability of detection due to bit sign transition

The losses on the non-centrality parameter considering one bit transition and no Doppler and code delay errors are represented in Fig. 5. This figure clearly shows that the effect of the sign transition can be important for a transition close to the center of the integration interval. This should induce a strong degradation of the probability of detection compared to the case without data bit sign transitions.

To conclude this section, the probability of detection for one integration $(K=1)$ knowing that a bit sign transition occurs at $t_{0}$ during the correlation interval is as follows:

$$
P_{d_{1, t_{0}}}=1-F_{\chi^{2}\left(2, \lambda_{t_{0}}\right)}\left(T_{h}\right)
$$

\section{Generalization of the Probability of Detection to Any} Number of Noncoherent Summations

The previous analysis can be extended over several noncoherent summations to give the general expression of the probability of detection. Knowing that over the dwell time $K T_{I}$, exactly, $N$ bit transitions occur (which can depend on the considered slice of received signal), the average probability of detection noted as $P_{d, t_{0}}$ is then:

$$
P_{d, t_{0}}=\sum_{n=0}^{N} P_{n} \times P_{d_{n, t_{0}}}
$$

where

- $P_{n}$ represents the probability of occurrence of $n$ bit sign transitions over the dwell time (which depends on $K$ and $\left.T_{I}\right)$

- $P_{d_{n, t_{0}}}$ represents the probability of detection knowing that $n$ bit sign transitions occur over the dwell time (and thus exactly $n$ correlator output pairs are affected by these transitions according to our assumptions). This quantity depends on $\varepsilon_{\tau}, \varepsilon_{f}, T_{I}, K$ and the location of transition $t_{0}$. It can be expressed as

$$
P_{d_{n, t_{0}}}=1-F_{\chi^{2}(2, \lambda)}\left(T_{h}\right)
$$

where $\lambda=n \times \lambda_{t_{0}}+(K-n) \times \lambda_{0}$

\section{Average Probability of Detection}

The randomness of the location of $t_{0}$ has an impact on the computation of the average probability of detection. Let us define the maximum number of bit transitions within the dwell time as follows:

$$
N_{\max }=\left\lceil\frac{K T_{I}}{T_{d}}\right\rceil
$$

If there can be several correlations per data bit, $T_{I} \leq T_{d}$, (for instance GPS L1 C/A), two cases have to be considered. For example, for GPS L1 C/A, for dwell time $K T_{I}=35 \mathrm{~ms}$, $N_{\max }=2$ : however, in $25 \%$ of the cases, only one bit sign transition can occur within the dwell time (when the second bit transition is in the interval $[35 ; 40] \mathrm{ms}$ ) and in $75 \%$ of the cases, two bit sign transitions can occur. The percentage of $N_{\text {max }}-1$ bit sign transitions is in fact given by the following:

$$
\alpha=N-\frac{K T_{I}}{T_{d}}=\left\{\frac{K T_{I}}{T_{d}}\right\}
$$

Taking this into account, the general expression of the probability of detection becomes:

$$
\begin{gathered}
\forall T_{I} \leq T_{d} \text { such as } \frac{T_{d}}{T_{I}} \in \mathbb{N}, \forall K \in \mathbb{N}^{*}, P_{d, t_{0}}= \\
\frac{\alpha}{2^{N-1}} \sum_{n=0}^{N_{\max }-1}\left(\begin{array}{c}
N_{\max }-1 \\
n
\end{array}\right) P_{d_{n, t_{0}}}+\frac{1-\alpha}{2^{N}} \sum_{n=0}^{N_{\max }}\left(\begin{array}{c}
N_{\max } \\
n
\end{array}\right) P_{d_{n, t_{0}}}
\end{gathered}
$$

where $P_{d_{n, t_{0}}}$, defined in (26), takes into account the number of correlations affected by a bit sign transition through the noncentrality parameter.

In (28) and (29), $P_{d_{n, t_{0}}}$ depends upon a number of factors, including the location of the bit sign transition $t_{0}$ and the Doppler and code delay errors ( $\varepsilon_{f}$ and $\varepsilon_{\tau}$ respectively) because of the acquisition bin size and they all affect the value of $\lambda$. It 
is thus better look at an average performance when taking into account these parameters.

To do so, it can be assumed that the Doppler frequency and the code delay uncertainties of the incoming signal in the correct acquisition bin are uniformly distributed. Taking the example of GPS L1 C/A and assuming correlation duration of $T_{I}=1 \mathrm{~ms}$, the size of one acquisition cell is $[-250 ; 250] \mathrm{Hz} \times[-0.25 ; 0.25]$ chip. Following this example, for a $C / N_{0}$ of $40 \mathrm{~dB}-\mathrm{Hz}$, and the context of a potential bit sign transition and no noncoherent summations Fig. 6 represents, the bit sign transition and no non-coherent summations:

- The actual probabilities of detection within the right cell (color coded from blue to red)

- The average probability of detection over the whole acquisition cell (in green).

GPS L1 C/A - $\mathrm{T}_{\mathrm{I}}=1 \mathrm{~ms}-\mathrm{C} / \mathrm{N}_{0}=40 \mathrm{~dB}-\mathrm{Hz}-$ No transition

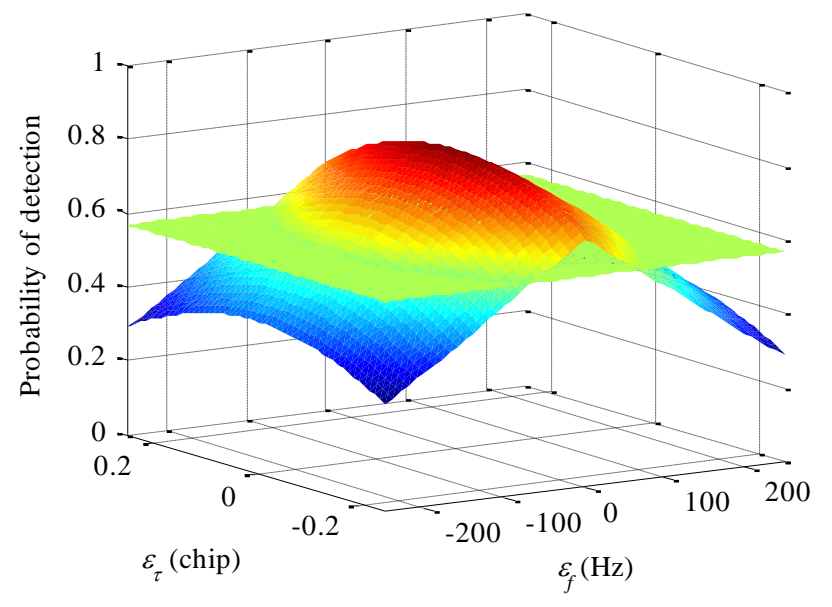

Fig. 6: Probabilities of detection for each bin in the right cell

Similarly, to assess the average effect of the bit sign transition on the probability of detection, it is proposed to take into account the location of the bit transition as a random variation. In the following, this random variable is assumed to be uniformly distributed over the data bit (or secondary code) duration. To conclude, the average probability of detection knowing that $n$ bit sign transitions occur over the dwell time is given by the following:

$$
\overline{P_{d}}=\mathrm{E}_{\text {correct cell }, t_{0}}\left[P_{d, t_{0}}\right]
$$

\section{APPLICATION TO GNSS SIGNALS}

To illustrate the previous generic results, two GNSS signals are studied as example. As presented in Section II.A, these signals exhibit quite different structures. In particular, Galileo E1 OS is representative of the new generation of GNSS signals.

\section{A. GPS LI C/A \\ 1) Optimal Acquisition Parameters}

To remain focused on a specific case (which represents reality), the correlation duration considered here does not exceed the data bit duration for GPS L1 C/A (20 ms). There are thus several choices for $T_{I}$.

Fig. 7. represents the case in which $1 \mathrm{~ms}$ correlations are used. Of more 20 successive 1-ms correlations, only 1 correlation can be affected by a data bit transition, which involves a bit sign transition with a probability of $50 \%$. The 19 other 1-ms correlations will necessarily be free of bit transitions.

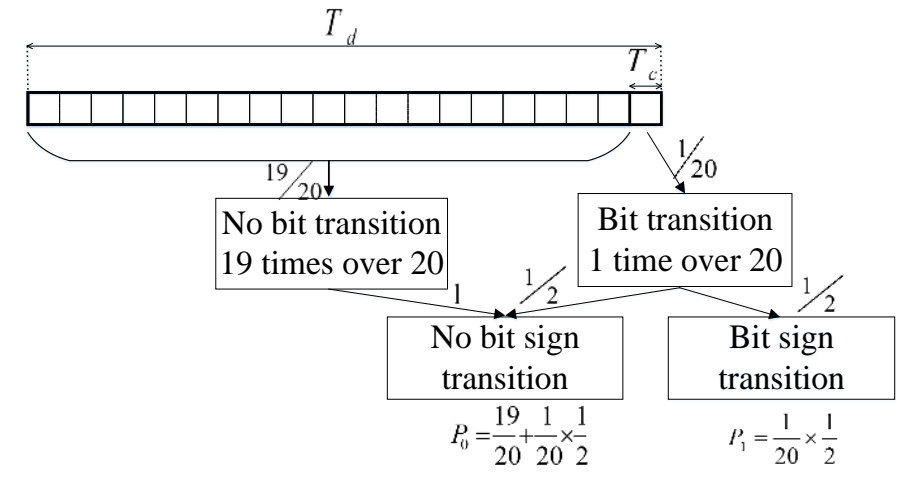

Fig. 7: Scheme to determine the probability of detection (GPS L1 C/A)

Based on (25), and for all possible $T_{I}$ such that $T_{I}$ is a multiple of the PRN code duration and $T_{I} \leq T_{d}$. TABLE II gives the average probability of detection $P_{d, t_{0}}$ for the simple case of $K=1$. It is a linear combination of the probability of 0 or 1 bit sign transition with:

$$
\begin{array}{r}
P_{d_{0, t_{0}}}=P_{d_{0}}=1-F_{\chi^{2}\left(2, \lambda_{0}\right)}\left(T_{h}\right) \\
P_{d_{1, t_{0}}}=1-F_{\chi^{2}\left(2, t_{0}\right)}\left(T_{h}\right)
\end{array}
$$

The parameters $\lambda_{0}$ and $\lambda_{t_{0}}$ depend on the coherent integration time $T_{I}$ as given in (11) and (18); thus $P_{d_{0}}$ and $P_{d_{1, t_{0}}}$ are different for each value of $T_{I}$.

TABLE II

AVERAGE PROBABILITY OF DETECTION FOR GPS L1 C/A FOR ONE INTEGRATION

\begin{tabular}{cc}
\hline \hline$T_{I}$ & $P_{d_{t_{0}}}=P_{0} \times P_{d_{0}}+P_{1} \times P_{d_{1, t_{0}}}$ \\
\hline $1 \mathrm{~ms}$ & $\left(\frac{19}{20}+\frac{1}{20} \times \frac{1}{2}\right) P_{d_{0}}+\left(\frac{1}{20} \times \frac{1}{2}\right) P_{d_{1, t_{0}}}$ \\
$2 \mathrm{~ms}$ & $\left(\frac{9}{10}+\frac{1}{10} \times \frac{1}{2}\right) P_{d_{0}}+\left(\frac{1}{10} \times \frac{1}{2}\right) P_{d_{1, t_{0}}}$ \\
$4 \mathrm{~ms}$ & $\left(\frac{4}{5}+\frac{1}{5} \times \frac{1}{2}\right) P_{d_{0}}+\left(\frac{1}{5} \times \frac{1}{2}\right) P_{d_{1, t_{0}}}$ \\
$5 \mathrm{~ms}$ & $\left(\frac{3}{4}+\frac{1}{4} \times \frac{1}{2}\right) P_{d_{0}}+\left(\frac{1}{4} \times \frac{1}{2}\right) P_{d_{1, t_{0}}}$ \\
$10 \mathrm{~ms}$ & $\left(\frac{1}{2}+\frac{1}{2} \times \frac{1}{2}\right) P_{d_{0}}+\left(\frac{1}{2} \times \frac{1}{2}\right) P_{d_{1, t_{0}}}$ \\
\hline $20 \mathrm{~ms}$ & $\frac{1}{2} P_{d_{0}}+\frac{1}{2} P_{d_{1, t_{0}}}$ \\
\hline
\end{tabular}

To illustrate TABLE II, Fig. 8 provides the average probability of detection for different $C / N_{0}$ values and for a dwell time of $20 \mathrm{~ms}$. The maximum average probability per $C / N_{O}$ is denoted with a star, allowing us to determine the optimal coherent integration time. As can be observed, the higher the $C / N_{0}$ is, the lower the coherent integration time. 


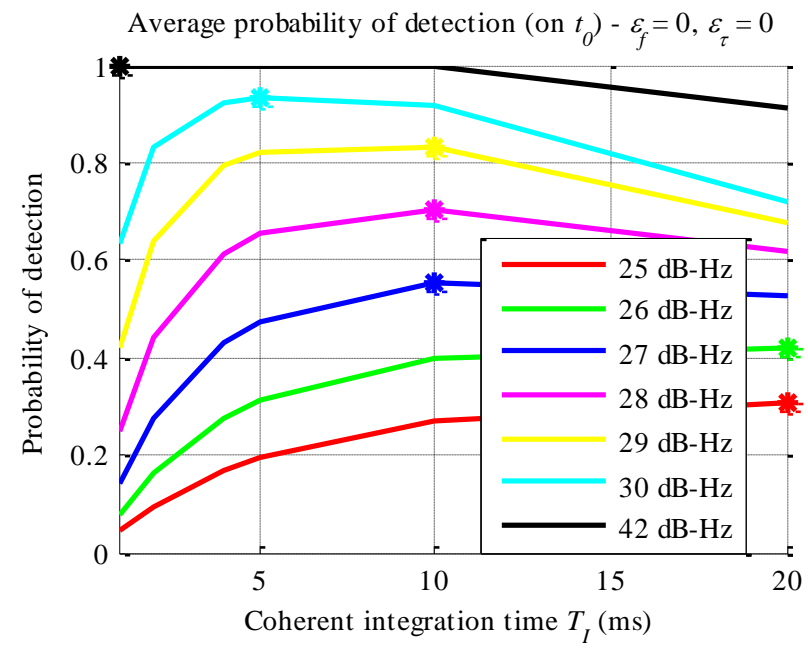

Fig. 8: Average probability of detection for different $C / N_{0}$ values, for a dwell time of $20 \mathrm{~ms}$

Based on (29), which is the generic expression of the average probability of detection, it is possible to determine via simulations the best couple $\left(K, T_{I}\right)$ to minimize the dwell time on one cell while ensuring a desired probability of detection. Taking as an example the objective to reach an average probability of detection of $95 \%$ for weak $C / N_{0}$ values, TABLE III gives the minimum dwell time $K T_{I}$ to reach the objective and the associated coherent integration time $T_{I}$ for which the probability of detection is the lowest above $95 \%$ using the following factors:

- The bit sign transition is not considered and there are no Doppler and code delay uncertainties (center of the right bin),

- The bit sign transition occurs in the worst location $\left(t_{0}=T_{I} / 2\right)$ and there are no Doppler and code delay uncertainties,

- A random location of the bit sign transition location, as described earlier, and there are no Doppler and code delay uncertainties.

The first column (dwell time $K T_{I}$ ) and second column (correlation duration $T_{I}$ ) for each case allow us to determine the corresponding number of noncoherent summations $K$.
TABLE III

OPTIMAL CORRELATION TIME TO REACH A PROBABILITY OF DETECTION OF 95\% (GPS L1 C/A) WITH $\varepsilon_{f}=0$ AND $\varepsilon_{\tau}=0$

\begin{tabular}{|c|c|c|c|c|c|c|}
\hline \multirow{2}{*}{$\begin{array}{c}C / \\
N_{0} \\
(\mathrm{~dB}- \\
\mathrm{Hz})\end{array}$} & \multicolumn{2}{|c|}{$\begin{array}{l}\text { No transition } \\
\text { considered }\end{array}$} & \multicolumn{2}{|c|}{$\begin{array}{c}\text { Bit sign } \\
\text { transition } \\
\text { considered at } \\
\text { worst location } \\
\left(t_{0}=T_{I} / 2\right)\end{array}$} & \multicolumn{2}{|c|}{$\begin{array}{l}\text { Bit sign transition } \\
\text { considered as } \\
\text { random }\end{array}$} \\
\hline & $\begin{array}{l}\text { Dwell } \\
\text { time } \\
(\mathrm{ms}) \\
\end{array}$ & $T_{I}(\mathrm{~ms})$ & $\begin{array}{l}\text { Dwell } \\
\text { time } \\
(\mathrm{ms}) \\
\end{array}$ & $\begin{array}{c}T_{I} \\
(\mathrm{~ms})\end{array}$ & $\begin{array}{l}\text { Dwell } \\
\text { time } \\
(\mathrm{ms})\end{array}$ & $\begin{array}{c}T_{I} \\
(\mathrm{~ms})\end{array}$ \\
\hline 25 & 60 & 20 & 120 & $5-10$ & 100 & 10 \\
\hline 26 & 40 & 20 & 85 & 5 & 80 & $5-10$ \\
\hline 27 & 40 & $10-20$ & 64 & 4 & 60 & $5-10$ \\
\hline 28 & 30 & 10 & 45 & 5 & 40 & 10 \\
\hline 29 & 20 & $10-20$ & 35 & 5 & 30 & 10 \\
\hline 30 & 20 & $\begin{array}{c}4-5- \\
10-20\end{array}$ & 25 & 5 & 24 & 4 \\
\hline 31 & 15 & 5 & 20 & $4-5$ & 20 & $4-5-10$ \\
\hline 32 & 10 & 10 & 15 & 5 & 15 & 5 \\
\hline 33 & 8 & 4 & 12 & $2-4$ & 10 & 5 \\
\hline
\end{tabular}

It can be seen that for low $C / N_{0}$ values, the optimal dwell time when considering the data bit sign transitions is twice that for when the data bit sign transitions are not considered. It can be also seen that the optimal acquisition parameters when bit sign transitions are considered differ significantly from the case in which they are not considered (which is often found in the literature or simplified to a considered $T_{I}$ of $1 \mathrm{~ms}$ ). The optimal values for $T_{I}$ depend on the targeted $C / N_{0}$. It is essential to consider the presence of data when deciding upon the acquisition parameters.

To correctly consider the previous results, it is important to put them in perspective. When dealing with acquisition performance, two criteria are critical: the mean acquisition time [15] and the sensitivity. In the context of this paper, we focus only on the sensitivity. However, the choice of the optimal coherent integration duration, as presented earlier also affects the number of Doppler and thus the time to explore the whole acquisition grid. As a consequence, the truly optimal coherent integration duration also has to be based on the mean acquisition time.

\section{2) Probability of Detection Considering Bit Sign Transitions and Doppler and Code Delay Residual Errors}

In the following, the specific case of a GPS L1 C/A signal received with a $C / N_{0}$ value of $27 \mathrm{~dB}-\mathrm{Hz}$ is studied. The objective is to understand the degradation of the probability of detection because of the joint effect of bit sign transitions, Doppler and code delay residual errors. Fig. 9 provides the average probability of detection taking into account the residual errors and bit sign transitions as a function of the dwell time for six values of the correlation duration $T_{I}$. Fig. 9 shows that for a $C / N_{0}$ value of $27 \mathrm{~dB}-\mathrm{Hz}$, the optimal correlation duration $T_{I}$ is $5 \mathrm{~ms}$ or $10 \mathrm{~ms}$. It appears that $T_{I}=5 \mathrm{~ms}$ is probably more adapted for a $C / N_{0}$ greater than 27 $\mathrm{dB}-\mathrm{Hz}$ and it is not as important to use long correlations. When looking at the actual probability of detection, it appears 
that the effect of the acquisition cell size is important: in the case of a $60 \mathrm{~ms}$ dwell time, the best probability of detection is $85 \%$ while Table III (not considering code delay and Doppler errors) showed $95 \%$.

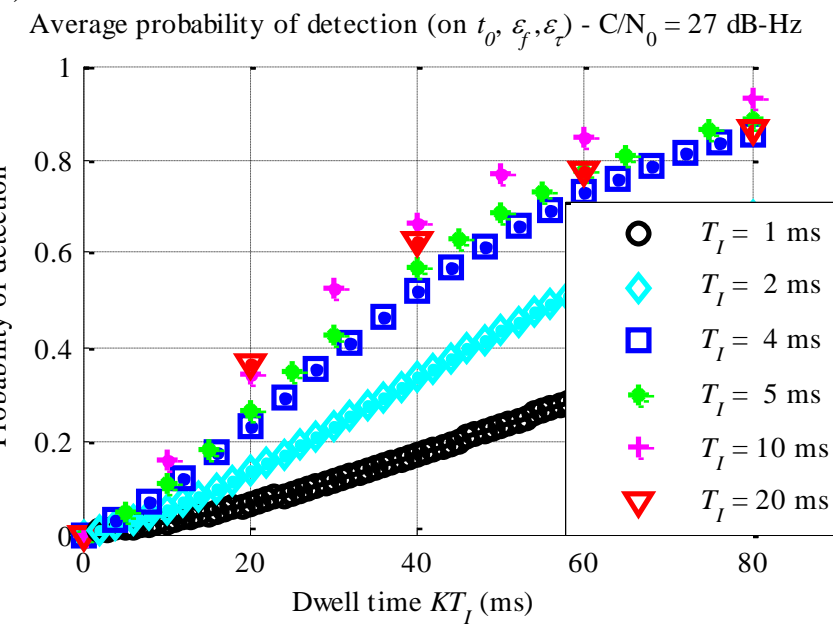

Fig. 9: Average probability of detection at $27 \mathrm{~dB}-\mathrm{Hz}$ for GPS L1 C/A (with bit sign transitions and residual code delay and Doppler errors)

For a high $C / N_{0}$, the effect of the data bit sign transition is not as pronounced because the optimal dwell time can be short (1 ms) and thus less affected by bit transitions.

In this paper, the theory has been developed considering that the coherent integration durations are multiples of the PRN code duration and dividers of the data bit duration. However, it is also possible to choose durations that do not divide the data bit duration, such as $6 \mathrm{~ms}$. In this case, the average probability of detection can be modeled based on the same theoretical methodology, however it is complex and we chose not to present it. Some elements to compute it are proposed using as an example a dwell time of $60 \mathrm{~ms}$. Let us assume that there is a shift of $1 \mathrm{~ms}$ between the local and the incoming signal:

- There is a bit transition at $t_{0}=1 \mathrm{~ms}$ during the first integration $[0,6] \mathrm{ms}$ (data bit transition at $1 \mathrm{~ms}$ ).

- There is a bit transition at $t_{0}=3 \mathrm{~ms}$ during the fourth integration $[18,24] \mathrm{ms}$ (data bit transition at $21 \mathrm{~ms}$ ).

- There is a bit transition at $t_{0}=5 \mathrm{~ms}$ during the seventh integration $[36,42] \mathrm{ms}$ (data bit transition at $41 \mathrm{~ms}$ )

However, if there are two bit sign transitions, the average probability of detection is not the same if they occur at the first and the second bit transitions or at the first and the third bit transitions. The average probability of detection should then take into account all the potential combinations of the locations of bit sign transitions (which depend on the correlation intervals).

\section{B. $\quad$ Galileo E1 OS Signal}

\section{1) Acquisition of Data and Pilot Signal Method}

For weak signals with data and pilot components such as Galileo E1 OS, it is preferable to acquire both components to avoid a loss of $3 \mathrm{~dB}$ on the received $C / N_{0}$. An acquisition method consists of considering the two components separately $[16,17]$. The data and pilot components of the received signal are thus correlated separately with the local spreading codes of the data and pilot components respectively.

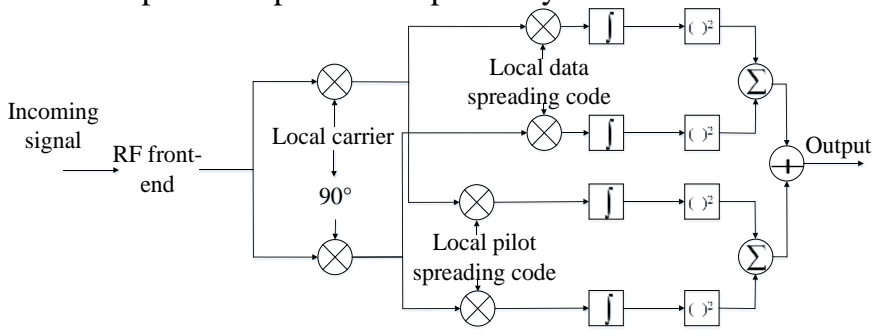

Fig. 10: Conceptual scheme of the data/pilot signal acquisition

The acquisition detector is then the sum of the two squared correlator outputs pairs corresponding to the data and pilot components as presented in Fig. 10.

$$
\begin{gathered}
T_{t_{0}}=\sum_{\mathrm{k}=0}^{\mathrm{K}} \frac{I_{t_{0}, \text { Data }}^{2}(k)}{\sigma^{2}}+\frac{Q_{t_{0}, \text { Data }}^{2}(k)}{\sigma^{2}}+\frac{I_{t_{0}, \text { Pilot }}^{2}(k)}{\sigma^{2}} \\
+\frac{Q_{t_{0}, \text { Pilot }}^{2}(k)}{\sigma^{2}}
\end{gathered}
$$

Assuming that the receiver uses the binary offset carrier BOC $(1,1)$ local replica on the data and pilot components, there is no distinction between the data autocorrelation function and the pilot one for Galileo E1 OS. Moreover, the crosscorrelation between the data and pilot spreading codes is assumed to be negligible because the choice of the spreading codes is as orthogonal as possible.

The duration of the spreading codes on Galileo E1 OS is equal to the duration of a data bit on the data component and to the duration of a secondary code bit on the pilot component. Therefore, a correlation duration equal to that of the spreading code period (4 ms) only is considered in this section.

\section{2) General Expression of the Probability of Detection for a Data/Pilot Signal}

From the described acquisition scheme, four correlator outputs have to be considered for the acquisition detector. This modifies the distribution of the acquisition detector: it remains a chi-square distribution, but with $4 K$ degrees of freedom. From the Galileo E1 OS signal structure, a bit sign transition (the data bit for the data component and the secondary code for the pilot component) can occur at every spreading code period on the data and pilot components with a probability of $50 \%$. Because the data bit duration is equal to the spreading code period (which implies $T_{I}=T_{d}$ ), the maximum number of bit sign transitions is $N_{\max }=2 K(\alpha=0)$. The probability of occurrence of $n$ bit sign transitions can be modeled by a binomial distribution $\mathcal{B}(2 K, 1 / 2)$ when $K$ noncoherent summations are used. The general expression of the average probability of detection, given by (29), can be simplified for Galileo E1 OS as:

$$
\forall K \in \mathbb{N}^{*}, \quad P_{d, t_{0}}=\frac{1}{2^{2 K}} \sum_{n=0}^{2 K}\left(\begin{array}{c}
2 K \\
n
\end{array}\right) P_{d_{n, t_{0}}}
$$

where $P_{d_{n, t_{0}}}=1-\mathcal{F}_{\chi^{2}\left(4 K, n \times \lambda_{t_{0}}+(2 K-n) \times \lambda_{0}\right)}\left(T_{h}\right)$

TABLE IV presents the optimal dwell time to reach a probability of detection equal or above 0.95 and at $27 \mathrm{~dB}-\mathrm{Hz}$ when there is no Doppler and code delay residual error. This table shows that the effect of data bit transitions on the Galileo 
E1 OS probability of detection is more pronounced than it is for GPS L1 C/A. This is because the correlation duration is constrained by the data bit rate, which is equal to the spreading code repetition rate. This means that on both the data and pilot channels, a sign transition can occur within each correlation interval.

TABLE IV

REQUIRED INTEGRATION TIME TO REACH A PROBABILITY OF DETECTION OF 95\% (GALILEO E1 OS) WITH $\varepsilon_{f}=0$ AND $\varepsilon_{\tau}=0$

\begin{tabular}{|c|c|c|c|}
\hline $\begin{array}{l}C / N_{0} \\
(\mathrm{~dB}-\mathrm{Hz})\end{array}$ & $\begin{array}{c}\text { No } \\
\text { transition } \\
(\mathrm{ms})\end{array}$ & $\begin{array}{c}\text { Bit sign } \\
\text { transition } \\
\text { considered } \\
\text { at worst } \\
\text { location } \\
\left(t_{0}=T_{I} / 2\right) \\
(\mathrm{ms}) \\
\end{array}$ & $\begin{array}{c}\text { Bit sign } \\
\text { transition } \\
\text { considered } \\
\text { as random } \\
(\mathrm{ms})\end{array}$ \\
\hline 25 & 172 & 580 & 436 \\
\hline 26 & 116 & 388 & 292 \\
\hline 27 & 80 & 264 & 200 \\
\hline 28 & 56 & 180 & 136 \\
\hline 29 & 40 & 128 & 96 \\
\hline 30 & 28 & 92 & 68 \\
\hline 31 & 20 & 64 & 48 \\
\hline 32 & 16 & 48 & 36 \\
\hline 33 & 12 & 36 & 28 \\
\hline
\end{tabular}

As a consequence, in a general case, the optimal dwell time to reach an average probability of detection of $95 \%$ when considering bit sign transition is 2.5 times longer than when bit sign transitions are not considered. In the worst case, when the bit sign transition is in the middle of the coherent integration interval, the optimal dwell time is multiplied by around 3.3. These remarks highlight that the bit sign transition highly penalizes the acquisition of Galileo E1 OS if a specific countermeasure is not put in place. Even if such a countermeasure is used, the minimum dwell time to acquire a signal with $C / N_{0}$ lower than $30 \mathrm{~dB}-\mathrm{Hz}$ is higher than that for GPS L1 C/A.

\section{3) Probability of Detection Considering Bit Sign Transitions and Doppler and Code Delay Residual Errors}

As previously done for GPS L1 C/A, the focus is now on the joint effect of bit sign transitions and Doppler and code delay residual errors for the acquisition of Galileo E1 OS components. Fig. 11 provides the probabilities of detection, for a $C / N_{0}$ of $27 \mathrm{~dB}-\mathrm{Hz}$ and a dwell time of $200 \mathrm{~ms}$, considering the ideal case in which there are no residual errors and there is the assumption of the absence of data (blue curve), considering residual errors on the frequency and time domain and absence of data (magenta curve) and finally the average probability of detection when considering bit sign transition and Doppler and code delay residual errors (red curve). As given in Table IV, at $27 \mathrm{~dB}-\mathrm{Hz}$, in $200 \mathrm{~ms}$, the average probability of detection (only on the bit sign transition location) is $95 \%$. However, because of the strong of the acquisition cell size, it becomes $78 \%$ when adding code delay and Doppler frequency residuals errors.

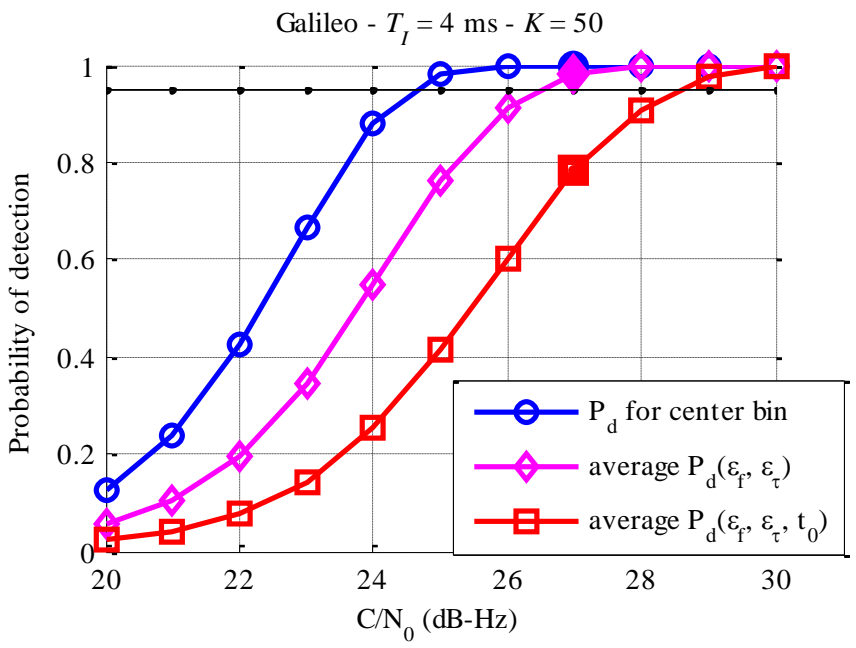

Fig. 11: Average probability of detection at $27 \mathrm{~dB}-\mathrm{Hz}$ for Galileo E1 OS

These results clearly indicate the need for acquiring Galileo E1 OS at low $C / N_{0}$ meaning that a specific acquisition technique resistant to data bit transitions has to be used. For example [18] and [19] tackle the problem of the acquisition degradations because of bit sign transitions, at the expense of a higher complexity. In [18], a data bit transition insensitive variant of the Double Block Zero Padding acquisition method is developed, dedicated to the modernized GNSS signals. This method is based on partial correlations. If the local and incoming blocks are exactly synchronized but the incoming signal is affected by a bit sign transition, the previous results for GPS L1 C/A are still true. Thus, the longer the coherent integration time (which means the spreading code period), the better is the results.

\section{CONCLUSIONS}

In this paper, a mathematical study of the impact of bit sign transitions on the acquisition performance was presented. First, developing the acquisition detection problem, the probability of detection considering bit sign transitions was expressed. The main result is that the acquisition degradation is maximum for a bit sign transition in the center of the correlation interval. In a general way, even if the transition is not at the worst location, the acquisition losses are not negligible and should be taken into account, when choosing the acquisition parameters.

Second, we determined the average probability of detection taking into account the Doppler and code delay uncertainties linked to the size of the acquisition bins. A uniform distribution of all of these errors within the bin was considered. The same work was presented to evaluate the average probability of detection considering bit sign transitions assuming a uniform distribution of the location of the bit sign transition.

Finally, two test cases were proposed: GPS L1 C/A and Galileo E1 OS. The first one has a single component and the second one is composed of a data component and a pilot component with a $50 \% / 50 \%$ power share. The mathematical development together with Monte-Carlo simulations showed that for the GPS L1 C/A signal, the optimal correlation time is generally 4 or $5 \mathrm{~ms}$ when considering the bit sign transitions, which allows good performance at high and low $C / N_{0}$ values. 
Also, these simulations showed that the total dwell time required to reach a high probability of detection can be strongly underevaluated (typically by a factor of 2) if the bit sign transition is not considered, which is usually the case in publications.

The structure of the Galileo E1 OS signal (a spreading sequence with a duration equal to that of the bit duration) is clearly detrimental to the acquisition performance. Although this can appear as a significant performance drawback, it only means that the acquisition technique used by a Galileo E1 OS receiver should also be insensitive to data bit transitions, which usually implies a more demanding processing. Such methods have been proposed in the literature are $[18,19]$.

\section{ACKNOWLEDGEMENTS}

The authors thank Jérôme Leclère from the

Electronics and Signal Processing Laboratory (ESPLAB) of the Ecole Polytechnique Fédérale de Lausanne (EPFL) for its comments and suggestions that greatly improved this article.

\section{REFERENCES}

[1] Navstar, "GPS Space Segment/Navigation User Interfaces (IS-GPS-200G).” Sep-2012.

[2] Navstar, "GPS Space Segment/User Segment L1C Interface (IS-GPS-800C).” Sep-2012.

[3] Navstar, "GPS Space Segment/User Segment L5 Interfaces (IS-GPS-705C)." Sep-2012.

[4] European Union, "European GNSS (Galileo) Open Service Signal In Space Interface Control Document (OS SIS ICD) Issue 1.2." Jun-2014.

[5] F. S. T. Van Diggelen, A-GPS: Assisted GPS, GNSS, and SBAS. Artech House, 2009.

[6] J. J. Parkinson and J. J. Spilker, Global Positioning System: Theory and Applications, American Institute of Aeronautics and Astronautics., vol. I, 2 vols. 1996.

[7] L. Lo Presti, X. Zhu, M. Fantino, and P. Mulassano, "GNSS Signal Acquisition in the Presence of Sign Transition," IEEE J. Sel. Top. Signal Process., vol. 3, no. 4, pp. 557-570, Aug. 2009.

[8] C. O'Driscoll, "Performance Analysis of the Parallel Acquisition of Weak GPS Signals," Ph.D. thesis, National University of Ireland, Cork (Ireland), 2007.

[9] M. Foucras, O. Julien, C. Macabiau, and B. Ekambi, "An Efficient Strategy for the Acquisition of Weak Galileo E1 OS Signals," in Proceedings of the European Navigation Conference 2013 (ENC 2013), Vienna (Austria), 2013.

[10] D. Borio, L. Camoriano, and L. Lo Presti, "Impact of GPS Acquisition Strategy on Decision Probabilities," IEEE Trans. Aerosp. Electron. Syst., vol. 44, no. 3, pp. 996 - 1011, Jul. 2008.

[11] J. Leclère, C. Botteron, and P.-A. Farine, "Comparison Framework of FPGA-based GNSS Signals Acquisition Architectures," IEEE Trans. Aerosp. Electron. Syst., vol. 49, no. 3, pp. 1497 - 1518, Jul. 2013.

[12] D. Borio, "GNSS Acquisition in the Presence of Continuous Wave Interference," IEEE Trans. Aerosp. Electron. Syst., vol. 46, no. 1, pp. 47 - 60, Jan. 2010.
[13] H. Al Bitar, "Advanced GPS Signal Processing Techniques for LBS Services," Ph.D. thesis, Institut National Polytechnique de Toulouse, 2007.

[14] RTCA, Inc, "Assessment of Radio Frequency Interference Relevant to the GNSS L1 Frequency Band RTCA/DO-235B." 13-Mar-2008.

[15] J. K. Holmes, Spread Spectrum Systems for GNSS and Wireless Communications. Artech House, 2007.

[16] D. Borio and L. Lo Presti, "Data and Pilot Combining for Composite GNSS Signal Acquisition," Int. J. Navig. Obs., vol. 2008, no. Article ID 738183, Mar. 2008.

[17] F. Bastide, O. Julien, C. Macabiau, and B. Roturier, "Analysis of L5/E5 Acquisition, Tracking and Data Demodulation Thresholds," in Proceedings of the 15th International Technical Meeting of the Satellite Division of The Institute of Navigation (ION GPS 2002), Portland, OR (USA), 2002, pp. 2196 - 2207.

[18] M. Foucras, O. Julien, C. Macabiau, and B. Ekambi, “A Novel Computationally Efficient Galileo E1 OS Acquisition Method for GNSS Software Receiver," in Proceedings of the 25th International Technical Meeting of The Satellite Division of the Institute of Navigation (ION GNSS 2012), Nashville, TN (USA), 2012, pp. $365-383$.

[19] K. Sun and L. L. Presti, "A Differential Post Detection Technique for Two Steps GNSS Signal Acquisition Algorithm," in Proceedings of IEEE/ION PLANS 2010, Indian Wells, CA, USA, 2010, pp. $752-764$.

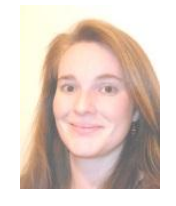

Myriam FOUCRAS received two M.Eng. degrees, one in Mathematical engineering and one in Fundamental Mathematics from the University of Toulouse in 2009 and 2010. She obtained her Ph.D. degree in 2015 from Ecole Nationale de l'Aviation Civile (ENAC) and funded by ABBIA GNSS Technologies, on the performance analysis of the modernized GNSS signals. In the same company, she continues her work and focuses on the performance of Galileo.

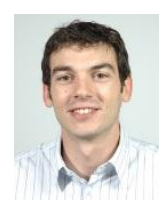

Olivier JULIEN is the head of the Signal Processing and Navigation (SIGNAV) research group of the TELECOM laboratory of ENAC, in Toulouse, France. His research interests are GNSS receiver design, GNSS multipath and interference mitigation and GNSS interoperability. He received his M.Eng. degree in 2001 in digital communications from the ENAC and his Ph.D. degreein 2005 from the Department of Geomatics Engineering of the University of Calgary, Canada.

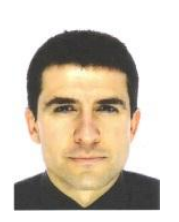

Christophe MACABIAU graduated with an M.Eng degree as an electronics engineer in 1992 from the ENAC in Toulouse, France. Since 1994, he has been working on the application of satellite navigation techniques to civil aviation. He received his $\mathrm{PhD}$. degree in 1997 and has been in charge of the 
TELECOM laboratory of the ENAC since 2011.

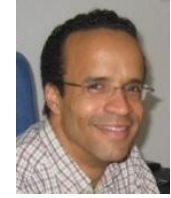

Bertrand EKAMBI graduated by a M.Eng. in Mathematical Engineering in 1999. Since 2000, he has involved in the main European GNSS projects: the European Geostationary Navigation Overlay Service (EGNOS) and Galileo. $\mathrm{He}$ is the founder manager of ABBIA GNSS Technologies, a French SME working on Space Industry project and based in Toulouse, France.

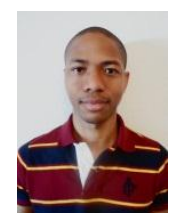

Fayaz BACARD received his M.S.E. specialized in Electronics and Computer Engineering from Ecole Nationale Supérieure des Sciences Appliquées et Technologies (ENSSAT) in Lanion, France in 2013. Since 2013, he has been a software engineer at ABBIA GNSS Technologies. 